\title{
Special Issue on Interfacial Bioengineering: Bridging the Gap From the Molecular Scale to the Macroscale
}

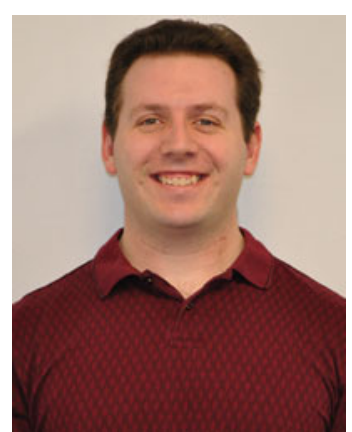

Michael Detamore
It is with great pleasure that I introduce to you the first special issue of a new era of the Annals of Biomedical Engineering under the leadership of Kyriacos Athanasiou. This special issue is more of a collection of articles sharing a common theme, rather than focusing on any one topic. The common theme is Interfacial Bioengineering, focusing on a number of bioengineering applications where an interface is a major design consideration. Some of the greatest advances in bioengineering have come at the interface, and it is the goal of this issue to capture some of these major advances in a collection of reviews and original articles across the spectrum of Interfacial Bioengineering. These invited contributions are categorized into eight topic categories, and provide an international flavor, with contributions coming from North America (United States), Europe
(Germany, Spain, and United Kingdom), and Asia (India, China, Taiwan, Japan, Korea, and Turkey).

The term "interface" can be defined in many ways. For this special issue, phase-boundary interfaces (e.g., gas-liquid, liquid-liquid) and "interfaces between disciplines" have been excluded, and instead the focus is on the interface between two or more different entities, spanning from the molecular level to the cellular level and macroscale. Examples of reports in this issue include reviews of the dentin-adhesive interface in dental composite restoration and biointerfaces for lab-on-a-chip devices, a comparison of electrode-skin interfaces, analyses of molecular interfaces and of hip implant interfaces, and interfacial tissue engineering in renal, vascular, neural, and orthopedic applications.

With the diversity of topics sharing a common theme with broad interest to the bioengineering community, this special issue captures the essence of the Annals of Biomedical Engineering, which strives to disseminate findings of broad interest and impact in our field. I hope you will enjoy this collection of articles exploring bioengineering at the interface. 